\title{
WCC 2016-121 : RARE CAUSES OF AORTIC VALVE INFECTIVE ENDOCARDITIS
}

K.Sridhar babu ,M Jyotsna, D Seshagiri Rao, Rama, Nlalitha.

\section{BACK GROUND}

Aortic valve infective endocarditis of Mycobacterium tuberculosis complex \& Brucella mellitensis are rare .we want to report two cases caused by them. Mycobacterium Tuberculosis have been observed at autopsy in the heart, pre-dominantly in the myocardium and endocardium, but rarely involving the coronary vessels , and valvular structures(1) . Mycobacterium tuberculosis valvular endocarditis is extremely rare, with most reports coming from autopsy series (2,3). Mycobacterium tuberculosis endocarditis has been reported only in the setting of miliary tuberculosis generally diagnosed postmortem.(4) Brucellosis is worldwide a zoonotic infective disease especially seen in developing countries. Frequently it is transmitted to humans through the consumption of products derived from unpasteurized milk and through direct contact with infected animal tissue(10).

\section{CASE PRESENTATION}

case no: 1

A 48 years old male patient presented with shortness of breath functional class -III since 15 days ,history of paraxysmal noctunal dyspnea present , atypical chest pain present ,h/o of low grade fever present since 10 days, no palpitations, no syncope.He is smoker \& alcoholic, stoped 3 yrs back.on examination nopallor,noclubbing,nocyanosis,nolymphadenopathy, Cvs - s1 soft PSM in mitral area, EDM in neoaortic area ECG -LVH2D ECHO -CRHD ,SEVERE AR,MILD AS, SEVERE MR,MRJA -10cm2 ,LV -7.4X5.4=58\% AVG -30/17,ARPHT-250 $\mathrm{m} / \mathrm{sec}$,mobile veg $0.5 \times 0.5 \mathrm{~cm}$ attached to AML, RCC of AORTIC valve $1 \times 0.5 \mathrm{~cm}$ (fig no: $1 \& 2$ ),Blood culture showed streptocccus sanguis Coronary angiogram showed normal coronaries , severe MR \& AR, DVR done after 1 month of antibiotic course, MVR -29mm ST JUDE MECHANICAL NON ROTATABLE VALVE,AVR-23mm ST JUDE MECHANICAL NON ROTATABLE VALVE.Post operative tissuse was sent for culture found to have positive for Mycobacterium tuberculosis complex after 7 weeks 


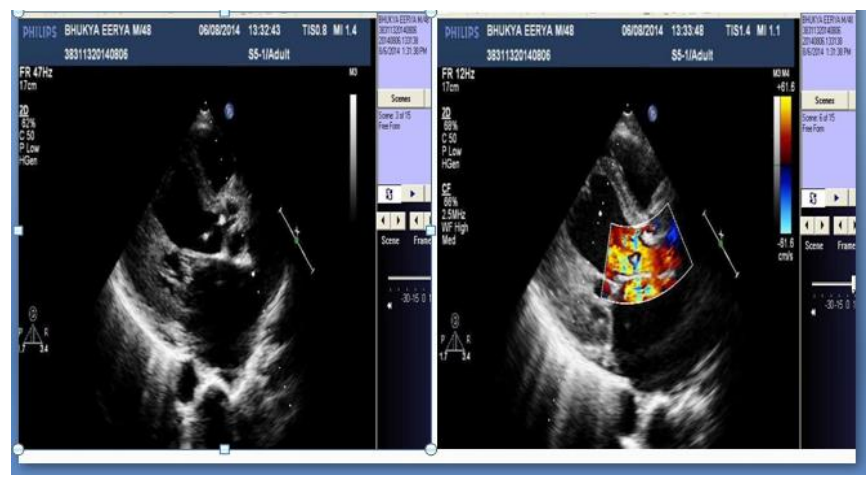

fig no: 1 aortic valve vegetation \&Doppler interrogation

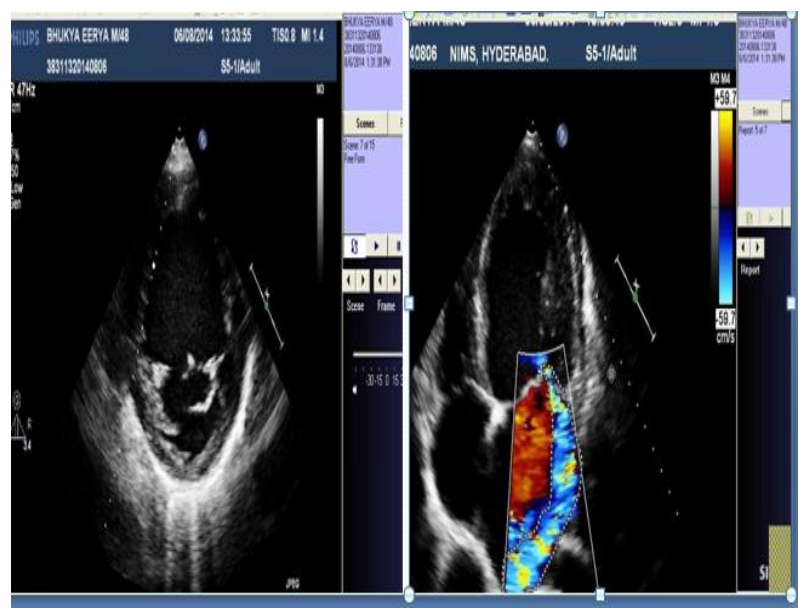

figno:2 mitral valve vegetation \& Doppler interrogation

\section{caseno:2}

A 36 year old male who is a shephereder by ocupation presented with high grade fever, exertional shortness of breath since 2 months,no orthopnea .on examination mild pallor, grade II clubbing, no jaundice, no lymphadenopathy,early diastolic murmur $>50 \%$ in neoarotic area ,short ejection systolic murmur in aortic area ,ECG - left ventricular hypertrophy ,chest x-ray mild cardiomegaly, 2Decho $-2 \mathrm{x} 1.1 \mathrm{~cm}$ sized vegetation on aoric valve with severe aortic regurgitation \& mild aortic stenosis,bicuspid aortic valve, good biventricular function(fig no:3,4).ESR-52mm/first hour,blood cultures showed growth for Brucella melitensis.patient fever subsided after starting on Doxycycline \& rifampicin . 


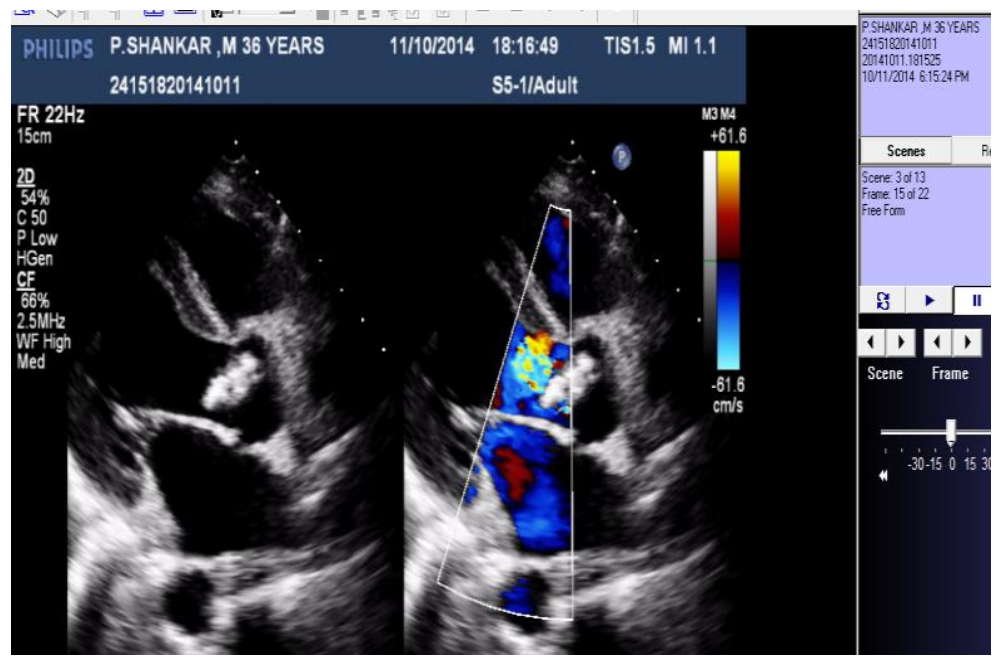

fig no:3 aortic valve vegetation \&doppler interogation

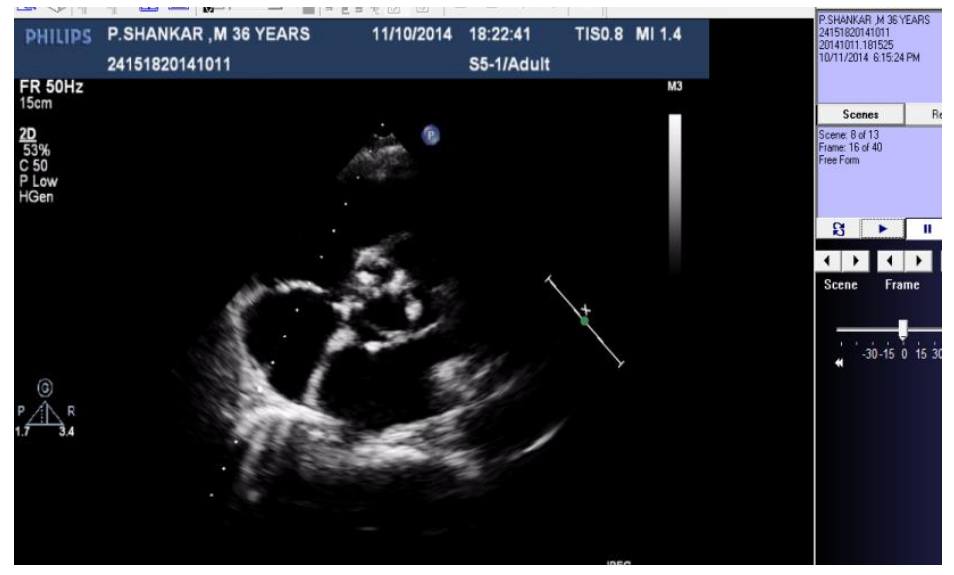

fig no:4 Bicuspid aortic valve with vegetation

\section{DISCUSSION}

We report a case of infective endocarditis along with severe aortic regurgitation and severe mitral regurgitation found to have Tuberculosis on tissue cultures of the vegetations.

Cardiac tuberculosis was recognized and described by Laennec in 1826, assigning the heart as the 13th organ affected in order of frequency [5]. Valvular endocarditis caused by $M$. Tuberculosis is extremely rare, and is reported only in the context of miliary tuberculosis. Cope et al. reported a case of disseminated tuberculosis with echocardiographically documented aortic valvulitis which resolved on antituberculous therapy [6]. Three cases of right sided tuberculous endocarditis in human immunodeficiency virus (HIV) positive intravenous drug abusers with disseminated tuberculosis have been observed. Endocardial miliary tubercles, polypoidal tubercles that resemble myxoma, nodules on valves, and thrombi containing entrapped tubercle bacilli have been described histopathologically. There are no reports in the literature of valvular 
endocarditis due to Mycobacterium tuberculosis without miliary tuberculosis in an immunocompetent patient. Another recent case has been reported of an infant with intracardiac tuberculoma in the background of miliary tuberculosis [7] Tuberculous mitral valve endocarditis and right atrial tuberculoma in an immunocompetent individual was reported(8). Recently anothercase of triple valve endocarditis by mycobacterium Tuberculosis in an immunocompetent host was reported(9) . Valvular endocarditis due to Mycobacterium tuberculosis in immunocompetent hosts should be added to the list of potential manifestations of Mycobacterium tuberculosis infections. Our patient did not manifest any involvement of the myocardium or pericardium although tuberculous pericarditis is a commoner clinical entity in this part of the world, the reasons for which are unclear.

Although brucella leads to many complications, cardiovascular involvement that is seen in less than $2 \%$ of cases usually manifests itself in clinical practice as endocarditis. Endocarditis is diagnosed lately in the course of the disease with mostly aorta valve involvement and leads to serious morbidity and mortality(10).

we report rare cause of aortic valve infective endocarditis which were diagnosed by prolon ged blood cultures,so if initial cultures are negative we should not neglect \& discard them because we will miss chance to diagnose $\&$ treart infective endocarditis caused by rare life threatening organisms . 